\title{
Is Routine Conventional Biopsy Sufficient for Thorough Histological Evaluation of a Neoplastic Lesion?
}

Science has come a long way since decades and centuries. It has reached its height, we think, but still it needs to go decades and centuries ahead. Development of science has reached different levels at different places. At one extreme science has started searching for possible settlement in another planet whereas at another extreme science has not at all been able to knock the door of poor people. Nepal is the country where probably the poorest of the poors live. Despite the fact that two giant neighbors of Nepal have developed science to its maximum height, Nepal has not even been able to peep through their development.

Among different branches of Medical science, histopathology is the one which opens the door to further treatment and which forecasts the possible outcome of a neoplastic lesion. The routine conventional procedure of histological evaluation in Nepal is what we commonly call "Biopsy" where we look basically for pathological features of a tumor such as benign or malignant cells, nuclear morphology, mitotic activities, necrosis, neovascularization etc. Accordingly we grade the lesion between grade I to grade IV on the basis of WHO system. This technique has been practiced for decades in Nepal. However, in developed world, including our neighborhood, advanced technology besides routine biopsy is regularly and thoroughly performed before finally giving the histopathological report.

At this modern stage of science and technology, is only routine biopsy sufficient for a thorough histological evaluation of a neoplastic lesion in Nepal? Do we really need something else, in addition, to know further about the tumor? If yes, is this the right time? Or shall we wait further?

Few questions that conventional biopsy can't answer are how fast the tumor is growing; what other prognostic histological markers are present in the tumor; if the tumor is grade III, is it grade III near to grade II or near to grade IV etc. Many a times, we come across similar experience where we can't answer properly the above questions and final outcome turns out to be exactly opposite to what we expect. In such situation it becomes too difficult to explain to patient and his relatives. Histology shows grade II tumor, but patient dies in two months, how come? Two different histological opinions for the same specimen, how come? Histology shows low grade tumor but it recurs very soon, how come? Therefore, don't we now need another technology which can answer these questions? Immunohistochemistry is the one.

Immunohistochemistry is a simple but a vital part of thorough histological evaluation. Without this, histological report of a neoplastic lesion is incomplete. It is a routine procedure in developed countries. Even in many south Asian countries it's a routine procedure or at least done whenever necessary. Many of the reagents needed for this are manufactured and easily available in India. So is in China. We import innumerable things for our daily life from these two countries, why not this technology? Cost factor is the biggest hurdle. But only a cost factor shouldn't prevent science to develop further. Antibodies are very expensive but $1 \mathrm{ml}$ of antibody is sufficient for hundreds of specimens. Another problem is if the antibody is not used in a fixed time period, usually six months to one year, it expires. However, once clinical practitioners are well aware of this technology, it will be so commonly used that antibody will be used up before it expires. Many of us seem to be unaware of this technology. The experts in this field need to guide and teach everybody to develop science and technology in this field in Nepal.

MIB I (Ki 67) is the universally known and scientifically proven proliferation marker of tumor. It can be used for neoplastic lesion of any organ of human body. ${ }^{1}$ With the information of MIB I index (proliferation index) we can predict the outcome of a lesion. Higher the MIB I index is, poorer is the prognosis 
Vascular endothelial growth factor (VEGF) is another marker which shows how vascular is the tumor and accordingly signifies poor prognosis if highly expressed. ${ }^{2}$ Glial fibrillay acidic protein (GFAP) is the diagnostic marker of glial brain tumor which can confirm the diagnosis and hence avoids confusion. ${ }^{3}$

Above are the few examples where immunohistochemistry can avoid diagnostic and prognostic dilemma. Hence, this is the right time that we should start doing immunohistochemistry in addition to conventional histological procedure to give proper diagnosis and treatment for the patients with tumors. It will help in development of oncology in Nepal.

\section{REFERENCES}

1. Oosterhuis JW, Schapers RF, Janssen-Heijnen ML, Smeets AW, Pauwels RP. RPMIB-1 as a proliferative marker in transitional cell carcinoma of the bladder: clinical significance and comparison with other prognostic factors. Cancer. 2000;88:2598-605.

2. Bajpai J, Sharma M, Sreenivas V, Kumar R, Gamnagatti S, Khan SA, et al. SVEGF expression as a prognostic marker in osteosarcoma. Pediatr Blood Cancer. 2009;53:1035-9.

3. Jung CS, Foerch C, Schänzer A, Heck A, Plate KH, Seifert V, et al. Serum GFAP is a diagnostic marker for glioblastoma multiforme. Brain. 2007;130:3336-41. 